\title{
Stable bound orbits in black lens backgrounds
}

\author{
Shinya Tomizawa ${ }^{1, *}$ and Takahisa Igata $\odot^{2, \dagger}$ \\ ${ }^{1}$ Mathematical Physics Laboratory, Toyota Technological Institute, Nagoya 468-8511, Japan \\ ${ }^{2}$ KEK Theory Center, Institute of Particle and Nuclear Studies, \\ High Energy Accelerator Research Organization, Tsukuba 305-0801, Japan
}

(Received 26 November 2020; accepted 14 December 2020; published 31 December 2020)

\begin{abstract}
In contrast to five-dimensional Schwarzschild-Tangherlini and Myers-Perry backgrounds, we show that there are stable bound orbits of massive and massless particles in five-dimensional black lens backgrounds, in particular, the supersymmetric black lens with $L(2,1)$ and $L(3,1)$ topologies. We also show that in the zero-energy limit of massless particles, there exist stable circular orbits on the evanescent ergosurfaces.
\end{abstract}

DOI: 10.1103/PhysRevD.102.124079

\section{INTRODUCTION}

A stable bound particle orbit is an orbit where a particle keeps moving in a bounded spatial region without reaching infinity or singularities even if small perturbations are applied. Such an orbit often appears in astrophysical phenomena because the stability results in a relatively long duration. The essence of the mechanism can be seen from the dynamics of a massive particle in the Schwarzschild spacetime. A massive particle moving on a stable bound orbit is localized near a radial potential well, which is made by a balance between the gravitational potential $-M m / r$ and the centrifugal potential $l^{2} /\left(2 m^{2} r^{2}\right)$, where $M$ is the black hole mass, $m$ and $l$ are the mass and angular momentum of a particle, respectively, and $r$ is the circumferential radius. The relativistic correction effect $-M l^{2} /$ $\left(m^{2} r^{3}\right)$ becomes dominated near the event horizon and causes proper relativistic phenomena such as perihelion shift and the innermost stable circular orbits. These can explain or predict events in the vicinity of black holes and other celestial objects, such as stellar orbital motion and accretion disks. Similarly, it was shown that stable bound orbits exist in the Kerr black hole spacetime [1].

For photons moving in the Schwarzschild spacetime and Kerr spacetime, it is very well known that there exist unstable circular orbits but not stable ones. However, in the Kerr-Newman spacetime with relatively large electric charge, stable photon orbits exist on the horizon [2], and in the 4D Majumdar-Papapetrou spacetimes with two black

\footnotetext{
tomizawa@toyota-ti.ac.jp

igata@post.kek.jp
}

Published by the American Physical Society under the terms of the Creative Commons Attribution 4.0 International license. Further distribution of this work must maintain attribution to the author(s) and the published article's title, journal citation, and DOI. Funded by SCOAP. holes, they appear even outside the horizon [3-5]. The existence of such stable bound photon orbits has a physically significant meaning because it implies instability of the background spacetime in the following sense: If they exist, many massless particles (not only photons but also gravitons, etc.) are stably trapped on the orbits and accumulate more and more in the finite region of the spacetime. This will cause so large backreaction to the background geometry that it will eventually break the background. On the other hand, from the wave perspective, linear waves localize in the vicinity of the trapping null geodesics resulting in a long timescale for the decay [6]. This phenomenon suggests the existence of nonlinear instabilities of the background spacetime [7].

It is now evident that even within the framework of vacuum Einstein gravity, there is a much richer variety of black hole solutions in higher dimensions. For instance, asymptotically flat, stationary and biaxisymmetric fivedimensional black holes can have three types of topologies of the event horizon, a sphere $S^{3}$, a ring $S^{1} \times S^{2}$ and lens spaces $L(p, q)$ [8-11]. The corresponding solutions are, respectively, called a black hole, a black ring [12-14] and a black lens $[15,16]$. For the Schwarzschild-Tangherlini solution and Myers-Perry solution in five dimensions, it was shown that there are no stable circular orbits in equatorial planes [17-21]. For the black ring solutions, it was shown in contrast to black holes that there exist stable bound orbits [22-24]. For the black lens solutions, in our previous work [25], we numerically show examples of stable bound orbits of particles around the supersymmetric black lens with the horizon topology of $L(2,1)$ in the fivedimensional minimal supergravity.

In this paper, focusing on the supersymmetric black lenses with $L(2,1)$ and $L(3,1)$ topologies in the fivedimensional minimal supergravity, we give more detail about the existence of the stable bound orbits for the massive and massless particles which move around the 
horizon than in the previous paper. In our analysis, we consider such motion of particles as a two-dimensional potential problem, where a problem of whether a stable bound orbit exists for massive or massless particles is reduced to a simple problem of whether the two-dimensional effective potential has a negative or zero local minimum. We also discuss if there are stable bound orbits at infinity because for the five-dimensional MajumdarPapapetrou solution with two black holes [26], they can exist at infinity when the separation of two black holes is large enough.

The rest of the paper is composed as follows: In the following Sec. II, we briefly review the supersymmetric black lens solution in the five-dimensional minimal supergravity. In Sec. III, we provide our formalism to show the existence of stable bound orbits. In Sec. IV, we show that there are stable bound orbits for supersymmetric black lenses with $L(2,1)$ and $L(3,1)$ topologies. In Sec. V, we summarize our results and discuss possible generalizations of our analysis to other black hole backgrounds.

\section{BLACK LENSES}

\section{A. Solutions}

In the five-dimensional minimal supergravity, the local metric and gauge potential 1-form of the supersymmetric black lens solutions take the form $[15,16]$

$$
\begin{gathered}
d s^{2}=-f^{2}(d t+\omega)^{2}+f^{-1} d s_{M}^{2}, \\
A=\frac{\sqrt{3}}{2}\left[f(d t+\omega)-\frac{K}{H}(d \psi+\chi)-\xi\right],
\end{gathered}
$$

where $d s_{M}^{2}$ is the Gibbons-Hawking metric, which is given by

$$
\begin{gathered}
d s_{M}^{2}=H^{-1}(d \psi+\chi)^{2}+H\left(d x^{2}+d y^{2}+d z^{2}\right), \\
\chi=\sum_{i=1}^{n} h_{i} \frac{z-z_{i}}{r_{i}} \frac{x d y-y d x}{x^{2}+y^{2}}, \\
H=\sum_{i=1}^{n} \frac{h_{i}}{r_{i}}:=\frac{n}{r_{1}}-\sum_{i=2}^{n} \frac{1}{r_{i}},
\end{gathered}
$$

where $r_{i}:=\left|\boldsymbol{r}-\boldsymbol{r}_{i}\right|=\sqrt{x^{2}+y^{2}+\left(z-z_{i}\right)^{2}}, \boldsymbol{r}:=(x, y, z)$ and $\boldsymbol{r}_{i}:=\left(0,0, z_{i}\right)$. For the constants $z_{i}$, we assume $z_{1}=$ $0<z_{2}<\cdots<z_{n}$ and $H$ is a harmonic function with point sources at $\boldsymbol{r}=\boldsymbol{r}_{i}$ on $\mathbb{E}^{3}$. The vectors $\partial / \partial t, \partial / \partial \psi$ and $\partial / \partial \phi:=x \partial / \partial y-y \partial / \partial x$ are Killing vectors, where $\partial / \partial \phi$ is the coordinate basis in the standard polar coordinates $(x=r \sin \theta \cos \phi, y=r \sin \theta \sin \phi, z=r \cos \theta)$. The other functions and 1 -forms are written as

$$
f^{-1}=H^{-1} K^{2}+L
$$

$$
\begin{gathered}
\omega=\omega_{\psi}(d \psi+\chi)+\hat{\omega} \\
\omega_{\psi}=H^{-2} K^{3}+\frac{3}{2} H^{-1} K L+M \\
\hat{\omega}=\sum_{j=2}^{n}\left[\frac{n}{2} k_{j}^{3}+\frac{3}{2}\left(k_{1} k_{j}^{2}-k_{j} l_{1}\right)\right] \frac{r-z_{j} \cos \theta}{z_{j} r_{j}} d \phi \\
+\sum_{i, j=2(i \neq j)}^{n}\left(-\frac{1}{2} k_{j}^{3}+\frac{3}{2} k_{i} k_{j}^{2}\right) \\
\times \frac{r^{2}-\left(z_{i}+z_{j}\right) r \cos \theta+z_{i} z_{j}}{z_{j i} r_{i} r_{j}} d \phi \\
-\frac{3}{2} \sum_{i=1}^{n}\left(-\sum_{j=1}^{n} k_{j} h_{i}+k_{i}\right) \frac{z-z_{i}}{r_{i}} d \phi \\
-\sum_{j=2}^{n} \frac{n k_{j}^{3}+3\left(k_{1} k_{j}^{2}-k_{j} l_{1}\right)}{2 z_{j}} d \phi-\sum_{i, j=2(i \neq j)}^{n} \frac{h_{i} k_{j}^{3}+3 k_{i} k_{j}^{2}}{2 z_{j i}} d \phi
\end{gathered}
$$

$$
\xi=-\sum_{i=1}^{n} k_{i} \frac{z-z_{i}}{r_{i}} d \phi
$$

where $z_{j i}:=z_{j}-z_{i}$ and

$$
\begin{gathered}
K=\sum_{i=1}^{n} \frac{k_{i}}{r_{i}}, \\
L=1+\frac{l_{1}}{r_{1}}+\sum_{i=2}^{n} \frac{k_{i}^{2}}{r_{i}}, \\
M=-\frac{3}{2} \sum_{i=1}^{n} k_{i}+\sum_{i=2}^{n} \frac{k_{i}^{3}}{2 r_{i}} .
\end{gathered}
$$

As discussed in Ref. [16], from the requirements of regularity at $\boldsymbol{r}=\boldsymbol{r}_{i}(i=2, \ldots, n)$ and the absence of closed timelike curves around the horizon $\boldsymbol{r}=\boldsymbol{r}_{1}$ and the $(n-1)$ points $\boldsymbol{r}=\boldsymbol{r}_{i}(i=2, \ldots, n)$, the parameters $\left(k_{i \geq 1}, l_{1}, z_{i \geq 2}\right)$ must satisfy

$$
\begin{aligned}
1+\frac{1}{z_{i}}\left(l_{1}\right. & \left.-2 k_{i} k_{1}-n k_{i}^{2}\right)+\sum_{j=2(j \neq i)}^{n} \frac{1}{\left|z_{j i}\right|}\left(k_{j}-k_{i}\right)^{2}<0, \\
- & \frac{3}{2} \sum_{j=1}^{n} k_{j}-\frac{3}{2} k_{i}+\frac{n k_{i}^{3}+3 k_{1} k_{i}^{2}-3 l_{1} k_{i}}{2 z_{i}} \\
& +\sum_{j=2(j \neq i)}^{n} \frac{\left(k_{j}-k_{i}\right)^{3}}{2\left|z_{j i}\right|}=0
\end{aligned}
$$


for $i=2, \ldots, n$ and the inequalities

$$
k_{1}^{2}+n l_{1}>0, \quad l_{1}^{2}\left(3 k_{1}^{2}+4 n l_{1}\right)>0 .
$$

It is shown in Ref. [16] that when the parameters simultaneously satisfy these conditions, the point $\boldsymbol{r}=\boldsymbol{r}_{1}(=0)$ denotes a null degenerate horizon whose spatial cross section is the lens space $L(n, 1)$, whereas the points $\boldsymbol{r}=\boldsymbol{r}_{i}(i=2, \ldots, n)$ give regular points, which correspond to the merely coordinate singularities like the origin of the Minkowski spacetime in the Gibbons-Hawking coordinates.

\section{B. Evanescent ergosurface}

The supersymmetric black lens admits the presence of evanescent ergosurfaces [15,16], which are defined as timelike hypersurfaces such that a stationary Killing vector field becomes null there and timelike everywhere except there. Reference [27] proved that on such surfaces, massless particles with zero energy $(E=0)$ relative to infinity move along stable trapped null geodesics. They exist at $f=0$, which corresponds to

$$
H=\sum_{i=1}^{n} \frac{h_{i}}{r_{i}}=0
$$

For $n=2$, they cross the points $z=2 z_{2} / 3$ and $z=2 z_{2}$ on the $z$ axis. For $n=3$, they cross the points $z$ satisfying

$$
F(z):=3\left|z-z_{2}\right|\left|z-z_{3}\right|-|z|\left|z-z_{3}\right|-|z|\left|z-z_{2}\right|=0
$$

on the $z$ axis. It turns out from simple computations that $F(z)=0$ has only a single root on $I_{+}$and $I_{1}$, two roots on $I_{2}$ and no root on $I_{-}$.

\section{New coordinates}

In the work of the geodesic motion of massive and massless particles around the black lenses, it is more convenient to use the coordinates $\left(\eta, \xi, \phi_{1}, \phi_{2}\right)$, defined by

$$
\begin{gathered}
\eta=2 \sqrt{r} \cos \frac{\theta}{2}, \quad \xi=2 \sqrt{r} \sin \frac{\theta}{2}, \\
\phi_{1}=\frac{\psi+\phi}{2}, \quad \phi_{2}=\frac{\psi-\phi}{2},
\end{gathered}
$$

than the Gibbons-Hawking coordinates $(r, \theta, \phi, \psi)$, where $\left(\phi_{1}, \phi_{2}\right)$ are the coordinates with $2 \pi$ periodicity. It should be noted that the points $\boldsymbol{r}_{i}=\left(0,0, z_{i}\right)(i=1,2, \ldots, n)$ on the $z$ axis correspond to $(\eta, \xi)=\left(\eta_{i}, 0\right)(i=1,2, \ldots, n)$ on the $\eta$ axis in the new coordinates, where $\eta_{i}:=2 \sqrt{z_{i}}$.

\section{D. $n=2$ case}

The case $n=2$ coincides with a black lens solution with the horizon topology of $L(2,1)$ in Ref. [15]. Equation (15) is simply written as

$$
z_{2}=\frac{k_{2}\left(3 k_{1} k_{2}+2 k_{2}^{2}-3 l_{1}\right)}{3\left(k_{1}+2 k_{2}\right)}(>0)
$$

and the inequalities (14) and (16) are, respectively,

$$
\begin{gathered}
1+\frac{l_{1}-2 k_{2} k_{1}-2 k_{2}^{2}}{z_{2}}<0, \\
l_{1}^{2}\left(3 k_{1}^{2}+8 l_{1}\right)>0 .
\end{gathered}
$$

The blue-shaded regions $\mathcal{D}$ in Fig. 1 show the parameter region where the inequalities (21)-(23) are simultaneously satisfied for $l_{1}=1$ under Eq. (21). In particular, we consider the case of $k_{1}=0$, which simplifies the conditions 23)-(21)) as

$$
z_{2}=\frac{2 k_{2}^{2}-3 l_{1}}{6}>0
$$

$$
1+\frac{l_{1}-2 k_{2}^{2}}{z_{2}}<0
$$

$$
l_{1}>0
$$

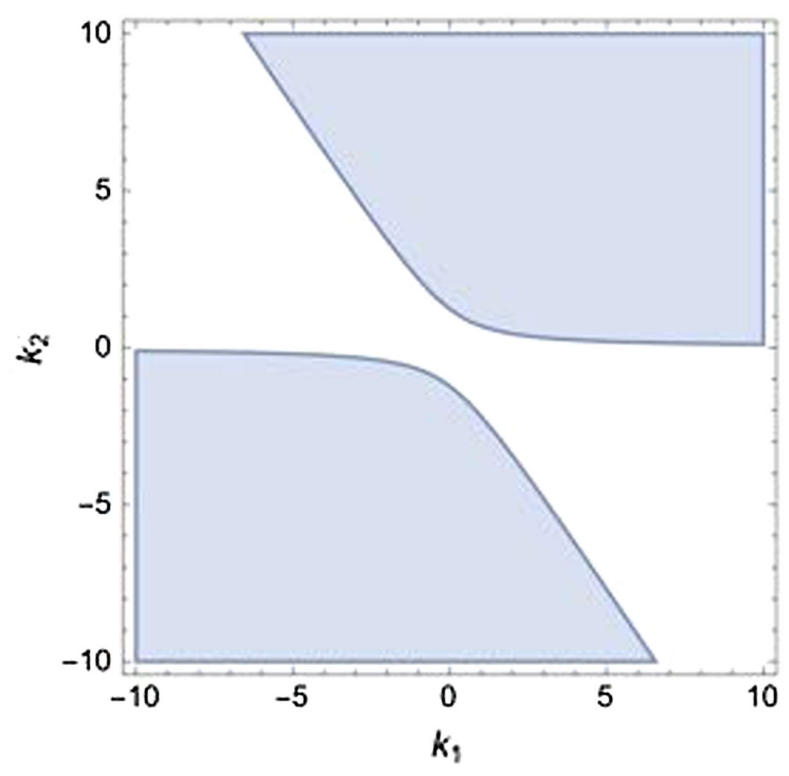

FIG. 1. Parameter regions $\mathcal{D}$ for the black lens with $L(2,1)$ topology. 


\section{E. $n=3$ case}

The case $n=3$ describes a black lens with the horizon topology of $L(3,1)$, which has four independent parameters $\left(k_{1}, k_{2}, k_{3}\right.$, and $\left.l_{1}\right)$. In this paper, for simplicity, we consider only the case of $k_{1}=0$, in which case the conditions (15) are written as

$$
\begin{aligned}
& -\frac{3}{2}\left(2 k_{2}+k_{3}\right)+\frac{3\left(k_{2}^{3}-l_{1} k_{2}\right)}{2 z_{2}}+\frac{\left(k_{3}-k_{2}\right)^{3}}{2 z_{32}}=0, \\
& -\frac{3}{2}\left(k_{2}+2 k_{3}\right)+\frac{3\left(k_{3}^{3}-l_{1} k_{3}\right)}{2 z_{3}}+\frac{\left(k_{2}-k_{3}\right)^{3}}{2 z_{32}}=0,
\end{aligned}
$$

and the inequalities (14) and (16) are reduced to, respectively,

$$
\begin{gathered}
1+\frac{l_{1}-3 k_{2}^{2}}{z_{2}}+\frac{\left(k_{3}-k_{2}\right)^{2}}{z_{32}}<0, \\
1+\frac{l_{1}-3 k_{3}^{2}}{z_{3}}+\frac{\left(k_{3}-k_{2}\right)^{2}}{z_{32}}<0, \\
l_{1}>0 .
\end{gathered}
$$

From Eqs. (27) and (28), $z_{2}$ and $z_{3}$ can be written as the functions of $k_{2}, k_{3}$ and $l_{1}$. When we normalize $l_{1}=1$ from (31), the inequalities (29) and (30) can be denoted by a certain region in a $\left(k_{2}, k_{3}\right)$ plane. This parameter region $\mathcal{D}$ which gives a black lens with $L(3,1)$ topology is drawn as the blue-colored region in Fig. 2.

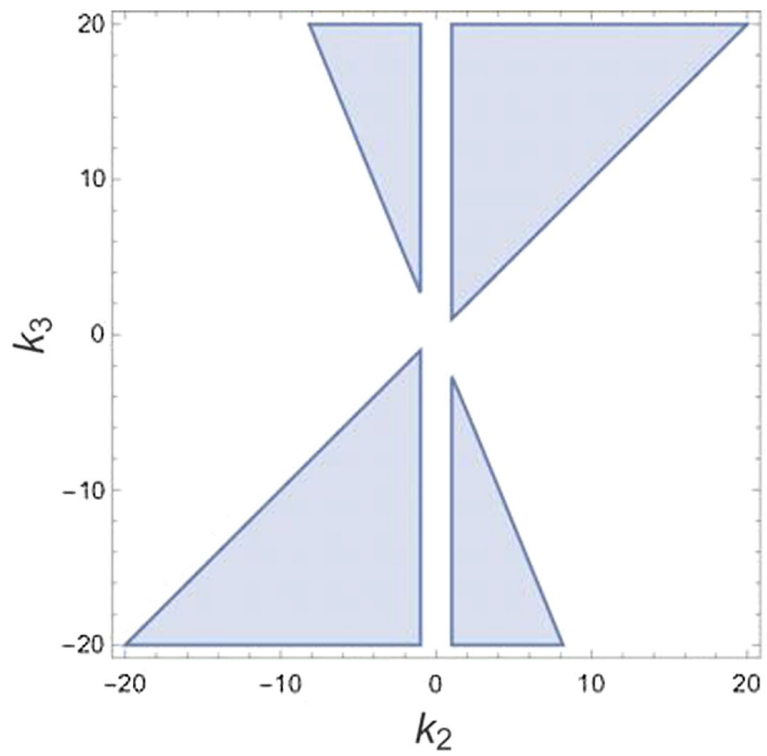

FIG. 2. Parameter regions $\mathcal{D}$ for the black lens with $L(3,1)$ topology $\left(k_{1}=0\right)$.

\section{OUR FORMALISM}

Our method to find stable bound orbits is based on the previous work [25], where we considered the geodesic motion of particles around the black lenses as a twodimensional potential problem. Now, we give the brief review as follows. The Hamiltonian of a free particle with the mass $m$ is written as

$$
\mathcal{H}=g^{\mu \nu} p_{\mu} p_{\nu}+m^{2},
$$

where $p_{\mu}$ is the momentum. From the independence of $\mathcal{H}$ on the coordinates $\left(t, \phi_{1}, \phi_{2}\right)$, the momenta $\left(p_{t}, p_{\phi_{1}}, p_{\phi_{2}}\right)$ are constants of motion, and we denote them as $\left(p_{t}, p_{\phi_{1}}\right.$, $\left.p_{\phi_{2}}\right)=\left(-E, L_{\phi_{1}}, L_{\phi_{2}}\right)$. Then, the Hamiltonian can be written in terms of these constants as

$$
\mathcal{H}=\frac{4 f}{H\left(\eta^{2}+\xi^{2}\right)}\left(p_{\eta}^{2}+p_{\xi}^{2}\right)+E^{2}\left(U+\frac{m^{2}}{E^{2}}\right) .
$$

The function $U$ is the effective potential defined by

$$
\begin{aligned}
U= & g^{t t}+g^{\phi_{1} \phi_{1}} l_{\phi_{1}}^{2}+g^{\phi_{2} \phi_{2}} l_{\phi_{2}}^{2}-2 g^{t \phi_{1}} l_{\phi_{1}}-2 g^{t \phi_{2}} l_{\phi_{2}} \\
& +2 g^{\phi_{1} \phi_{2}} l_{\phi_{1}} l_{\phi_{2}} \\
= & \frac{1}{4\left(K^{2}+H L\right)}\left[-3 K^{2} L^{2}+8 K^{3} M+12 H K L M\right. \\
& -4 H L^{3}+4 H^{2} M^{2}+\left(4 K^{3}+6 H K L\right. \\
& \left.\left.+4 H^{2} M\right)\left(l_{\phi_{1}}+l_{\phi_{2}}\right)+H^{2}\left(l_{\phi_{1}}+l_{\phi_{2}}\right)^{2}\right] \\
& +\frac{\left[-2 \hat{\omega}_{\phi}+\left(l_{\phi_{1}}+l_{\phi_{2}}\right) \chi_{\phi}+\left(l_{\phi_{2}}-l_{\phi_{1}}\right)\right]^{2}}{\left(K^{2}+H L\right) \eta^{2} \xi^{2}},
\end{aligned}
$$

where we have normalized two angular momenta by the energy as $l_{\phi_{1}}:=L_{\phi_{1}} / E$ and $l_{\phi_{2}}:=L_{\phi_{2}} / E$. Thus we can consider that the massive and massless particles move on the two-dimensional space $(\eta, \xi)$ while satisfying the Hamiltonian constraint $\mathcal{H}=0$. When we consider that the particles move in the two-dimensional potential $U$, the allowed regions of the motions for massive and massless particles are restricted to $U \leq-m^{2} / E^{2}$ and $U \leq 0$, respectively. From the determinant and trace of the Hesse matrix $\left(H_{i j}\right):=\left(U_{, i, j}\right)(i, j=\eta, \xi)$ of $U$, we can discuss the existence of a local minimum. If $\operatorname{Tr}\left(H_{i j}\right)>0$ and $\operatorname{det}\left(H_{i j}\right)>0$ at a stationary point $U_{, i}=0, U$ has a local minimum at the point.

For simplicity, we focus on the shape of the potential $U$ on the $z$ axis (i.e., $\theta=0, \pi$ ) of $\mathbb{E}^{3}$ in the Gibbons-Hawking space, which corresponds to $\eta=0$ and $\xi=0$ in the coordinates $(\eta, \xi)$. The $z$ axis is composed of the $n+1$ intervals: $\quad I_{-}=\{(\eta, \xi) \mid \eta=0, \xi>0\}, \quad I_{i}=\left\{(\eta, \xi) \mid \eta_{i}<\right.$ $\left.\eta<\eta_{i+1}, \xi=0\right\}(i=1,2, \ldots, n-1)$ and $I_{+}=\{(\eta, \xi) \mid \eta>$ $\left.\eta_{n}, \xi=0\right\}$. On $I_{+}$and $I_{-}$, only the particles with the angular momenta of $l_{\phi_{2}}=0$ and $l_{\phi_{1}}=0$, respectively, are 
allowed to stay there, whereas on $I_{i}(i=1, \ldots n-1)$ only the particles with the angular momenta of the special ratio of $l_{\phi_{1}} / l_{\phi_{2}}=-(n-i+1) /(n-i)$ are allowed to stay there because $I_{i}$ corresponds to the fixed points of the Killing isometry $\quad v:=(n-i) \partial / \partial \phi_{1}+(n-i+1) \partial / \partial \phi_{2}, \quad$ and hence only the particle with a zero angular momentum of $J:=p_{\mu} v^{\mu}=(n-i) L_{\phi_{1}}+(n-i+1) L_{\phi_{2}}=0$ can stay on the axis $I_{i}$ and otherwise the potential diverges on $I_{i}$.

In the above formalism, we must remove the zero-energy limit $E \rightarrow 0$ because $l_{\phi_{1}}$ and $l_{\phi_{2}}$ are divided by $E$. For this limit, it is better to use the potential $U^{\prime}$ defined by

$$
U^{\prime}=g^{\phi_{1} \phi_{1}} L_{\phi_{1}}^{2}+g^{\phi_{2} \phi_{2}} L_{\phi_{2}}^{2}+2 g^{\phi_{1} \phi_{2}} L_{\phi_{1}} L_{\phi_{2}}
$$

instead of the potential $U$, though in the previous work [25], we considered the zero energy as the limit $l_{\phi_{1}}, l_{\phi_{2}} \rightarrow$ $\pm \infty$ of $U$. The potential $U^{\prime}$ takes a simple form of

$U^{\prime}=\frac{H^{2}\left(L_{\phi_{1}}+L_{\phi_{2}}\right)^{2}}{4\left(K^{2}+H L\right)}+\frac{\left[\left(L_{\phi_{2}}-L_{\phi_{1}}\right)+\left(L_{\phi_{2}}+L_{\phi_{1}}\right) \chi_{\phi}\right]^{2}}{\left(K^{2}+H L\right) \eta^{2} \xi^{2}}$.

For this zero-energy limit, massive particles are not allowed to move because $U^{\prime}$ is non-negative, whereas massless particles can move on $U^{\prime}=0$, which corresponds to the intersection of two curves in the $(\eta, \xi)$ plane, $H=0$ and $G:=\left(L_{\phi_{2}}-L_{\phi_{1}}\right)+\left(L_{\phi_{2}}+L_{\phi_{1}}\right) \chi_{\phi}=0$, because the first and second terms in Eq. (37) are non-negative. It turns out hence that massless particles with zero energy always move on the evanescent surfaces, which correspond to $H=0$. Moreover, $U^{\prime}=0$ corresponds to a stationary point because at the point $\partial_{i} U^{\prime}=0(i=\eta, \xi)$ also holds.

It can be shown that $U^{\prime}$ has a local minimum at such a stationary point. To this end, let us confirm both the determinant and trace of the Hesse matrix $H:=$ $\left(\nabla_{i} \nabla_{j} U^{\prime}\right)(i, j=\eta, \xi)$ are positive at the stationary point, where $\nabla_{i}$ are the covariant derivatives associated with the two-dimensional conformally flat metric $g_{i j}$ in Eqs. (32) and (33). At just a stationary point such that $\nabla_{i} U^{\prime}=$ $\partial_{i} U^{\prime}=0, \nabla_{i} \nabla_{j} U=\partial_{i} \partial_{j} U^{\prime}$ can be shown because

$$
\begin{aligned}
\nabla_{i} \nabla_{j} U^{\prime}= & \partial_{i} \partial_{j} U^{\prime}-\Omega^{-1}\left(\partial_{i} U^{\prime} \partial_{j} \Omega+\partial_{j} U^{\prime} \partial_{i} \Omega\right. \\
& \left.-\delta_{i j} \partial_{k} U^{\prime} \partial^{k} \Omega\right),
\end{aligned}
$$

where $\Omega^{2}:=H\left(\eta^{2}+\xi^{2}\right) / 4 f$, so that it is enough to compute the determinant and trace of $\partial_{i} \partial_{j} U$, which are written, respectively, as

$$
\begin{aligned}
\operatorname{det} & \left.\left(\partial_{i} \partial_{j} U^{\prime}\right)\right|_{H=G=0} \\
= & U_{\eta \eta}^{\prime} U_{\xi \xi}^{\prime}-\left.U_{\eta \xi}^{\prime 2}\right|_{H=G=0} \\
= & \frac{\left(L_{\phi_{1}}+L_{\phi_{2}}\right)^{2}\left(H_{, \xi} G_{, \eta}-H_{, \eta} G_{, \xi}\right)^{2}}{K^{4} \eta^{2} \xi^{2}} \\
= & \frac{\left(L_{\phi_{1}}+L_{\phi_{2}}\right)^{4}\left(H_{, \xi}^{2}+H_{, \eta}^{2}\right)^{2}}{4 K^{4}}>0,
\end{aligned}
$$

$$
\begin{aligned}
& \left.\operatorname{Tr}\left(\partial_{i} \partial_{j} U^{\prime}\right)\right|_{H=G=0} \\
& =U_{\eta \eta}^{\prime}+\left.U_{\xi \xi}^{\prime}\right|_{H=G=0} \\
& =\frac{4\left(G_{, \eta}^{2}+G_{, \xi}^{2}\right)+\left(L_{\phi_{1}}+L_{\phi_{2}}\right)^{2}\left(H_{, \eta}^{2}+H_{, \xi}^{2}\right) \eta^{2} \xi^{2}}{2 K^{2} \eta^{2} \xi^{2}} \\
& =\frac{\left(L_{\phi_{1}}+L_{\phi_{2}}\right)^{2}\left(H_{, \eta}^{2}+H_{, \xi}^{2}\right)}{K^{2}}>0
\end{aligned}
$$

where we have used $G_{, \eta}=-\left(L_{\phi_{1}}+L_{\phi_{2}}\right) H_{, \xi} \eta \xi / 2$ and $G_{, \xi}=\left(L_{\phi_{1}}+L_{\phi_{2}}\right) H_{, \eta} \eta \xi / 2$, which are derived from $d \chi=*_{3} d H\left(\chi_{\phi, \eta}=-H_{, \xi} \eta \xi / 2, \chi_{\phi, \xi}=H_{, \eta} \eta \xi / 2\right)$.

For the particles with $J=0$ and $\left(L_{\phi_{1}}, L_{\phi_{2}}\right) \neq(0,0)$, one of the two conditions, $G=0$, is always satisfied at least on $I_{ \pm}$and $I_{i}(i=1, \ldots, n-1)$ because $\chi_{\phi}= \pm 1$ on $I_{ \pm}$and $\chi_{\phi}=2 n-2 i+1$ on $I_{i}(i=1,2, \ldots, n-1)$. Therefore, for such particles with zero energy, $U^{\prime}$ has a zero local minimum at the intersection of the evanescent ergosurfaces and the axes $I_{ \pm}$and $I_{i}(i=1, \ldots, n-1)$. This is why on such surfaces, massless particles with zero energy move along stable trapped null geodesics. In the following sections, we will discuss it from the contours of $U^{\prime}$ in the $(\eta, \xi)$ plane.

\section{STABLE BOUND ORBITS}

\section{A. A black lens with the topology $L(2,1)$}

In the previous work, we have seen the existence of stable bound orbits for the black lens with $L(2,1)$ topology, where we have focused on the behavior of the effective potential $U$ on the $z$ axis of $\mathbb{E}^{3}$ in the Gibbons-Hawking space. The $z$ axis is composed of the three intervals: $I_{-}=\{(\eta, \xi) \mid \eta=0, \xi>0\}, \quad I_{1}=\left\{(\eta, \xi) \mid \eta_{1}(=0)<\eta<\right.$ $\left.\eta_{2}, \xi=0\right\}$ and $I_{+}=\left\{(\eta, \xi) \mid \eta>\eta_{2}, \xi=0\right\}$. On $I_{+}$and $I_{-}$, only the particles with the angular momenta of $l_{\phi_{2}}=0$ and $l_{\phi_{1}}=0$, respectively, are allowed to stay there, whereas only ones with the angular momenta of the ratio $l_{\phi_{1}} / l_{\phi_{2}}=-2$ are allowed to stay on $I_{1}$.

\section{1. $I_{+}$}

First, we begin to comment on the existence of stable bound orbits on $I_{+}$. Figure 3 shows the typical shape of the effective potential $U$ with a negative local minimum on $I_{+}$, where we plot $U$ under the parameter setting $\left(k_{1}, k_{2}, l_{1}\right)=$ $(0,10,1)$ and the angular momenta $\left(l_{\phi_{1}}, l_{\phi_{2}}\right)=(-400,0)$. The left figure shows the shape of the effective potential on 

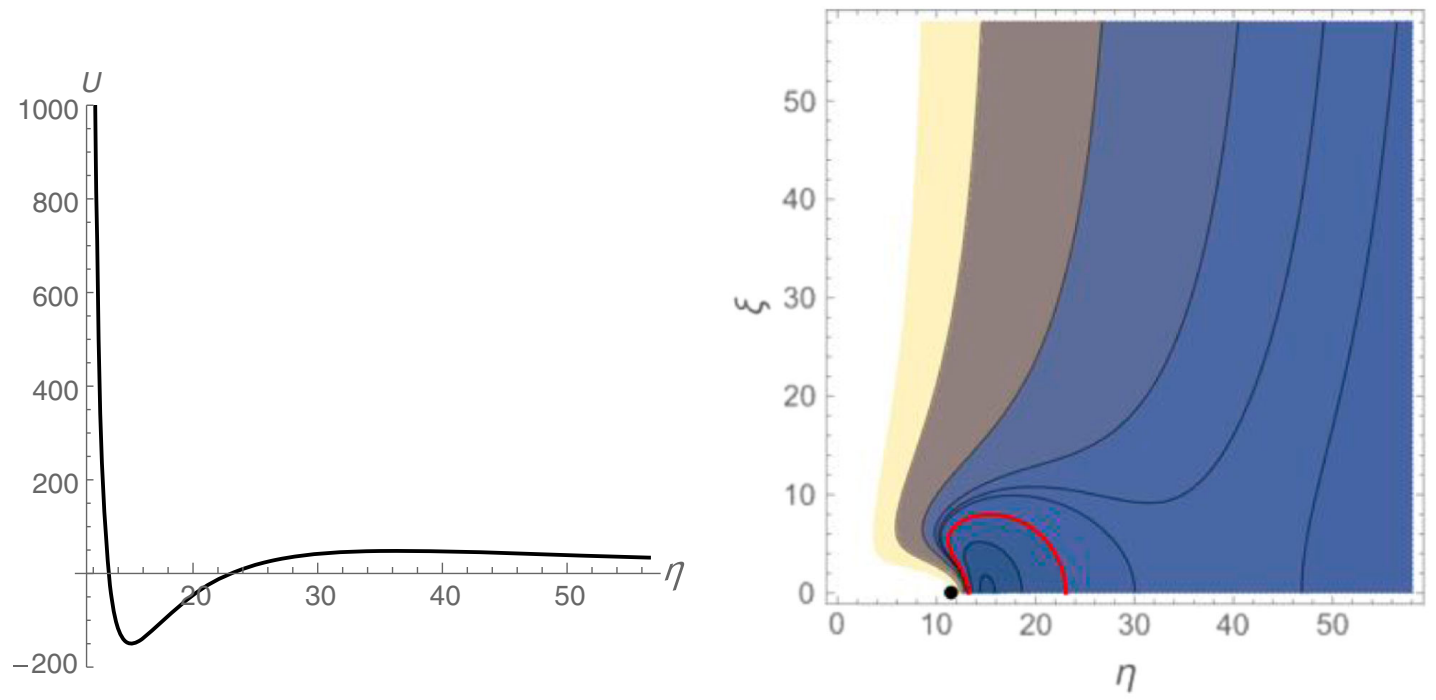

FIG. 3. The left figure shows the effective potential $U$ on $I_{+}\left(\eta>\eta_{2}=11.4 \ldots, z>z_{2}=32.8 \ldots\right)$ for $\left(k_{1}, k_{2}, l_{1}\right)=(0,10,1)$ and $\left(l_{\phi_{1}}, l_{\phi_{2}}\right)=(-400,0)$. The right figure shows the two-dimensional plot of $U$ and the red curve corresponds to $U=0$.

$I_{+}$, which is the intersection of $U$ by $\xi=0\left(\eta_{2}<\eta\right)$. The right figure shows the contours of $U$ for the particles with the same angular momenta. The red curve in this figure, which corresponds to $U=0$, separates the two-dimensional $(\eta, \xi)$ plane into two regions, the outer region $U>0$ and the inner region $U<0$. As can be read off from the contours, $U$ has a negative local minimum at a certain point $(\eta, \xi)=\left(\eta_{\mathrm{m}}, 0\right)$ on the $\eta$ axis $\left(z=z_{\mathrm{m}}\right.$ on the $z$ axis), where $\eta_{\mathrm{m}}\left(=2 \sqrt{z_{\mathrm{m}}}\right)$ is a certain constant satisfying $\eta_{2}<\eta_{\mathrm{m}}$. At the point $U_{, \eta}=U_{, \xi}=0, \operatorname{det}\left(H_{i j}\right)>0$ and $\operatorname{Tr}\left(H_{i j}\right)>0$. Therefore, both massive and massless particles inside the red curve are stably bounded in the finite region $U \leq$ $-m^{2} / E^{2}$ and $U \leq 0$, respectively. This means that there are stable bound orbits for massive and massless particles.

Furthermore, we consider whether there can exist such stable bound orbits at infinity $z \rightarrow \infty(\eta \rightarrow \infty, \xi=0)$ on $I_{+}$. To this end, let us expand the potential $U$ at $z \rightarrow \infty$ on $I_{+}$as

$$
U \simeq-1+\frac{U_{\infty}^{(1)}}{z}+\frac{U_{\infty}^{(2)}}{z^{2}}
$$

where the constants $U_{\infty}^{(1)}$ and $U_{\infty}^{(2)}$ are denoted, respectively, by

$U_{\infty}^{(1)}=\frac{l_{\phi_{1}}^{2}-16 k_{2}^{2}-8 l_{1}}{4}$,

$U_{\infty}^{(2)}=\frac{-\left(14 k_{2}^{2}+3 l_{1}\right) l_{\phi_{1}}^{2}+96 k_{2}^{3} l_{\phi_{1}}-160 k_{2}^{4}-24 l_{1}^{2}}{24}$.

After simple computations, we find that $0<-U_{\infty}^{(1)} \ll 1$ and $U_{\infty}^{(2)}>0$ are the necessary conditions that $U$ has a local minimum at infinity because the local minimum is at $z \simeq-2 U_{\infty}^{(2)} / U_{\infty}^{(1)}$. Noting that the condition $0<-U_{\infty}^{(1)} \ll 1$ can be denoted by $l_{\phi_{1}}= \pm 2 \sqrt{2\left(2 k_{2}^{2}+l_{1}\right)} \mp \epsilon(0<$ $\epsilon \ll 1)$, we can confirm that the leading term of $U_{\infty}^{(2)}$ can be written as

$$
\begin{aligned}
U_{\infty}^{(2)} \simeq & -\frac{2}{3}\left(24 k_{2}^{4}+10 l_{1} k_{2}^{2}+3 l_{1}^{2} \mp 12 k_{2}^{3} \sqrt{2\left(2 k_{2}^{2}+l_{1}\right)}\right) \\
& +\mathcal{O}(\epsilon) .
\end{aligned}
$$

From the leading terms, we find that $U_{\infty}^{(2)}<0$, which cannot satisfy the other condition $U_{\infty}^{(2)}>0$. As a result, there exist no stable bound orbits in the asymptotic region $z \rightarrow \infty$, at least, on the $z$ axis. This suggests that there exists the outermost stable circular orbit as the boundary of stable circular orbits.

\section{2. $I_{-}$}

Next, we focus on the potential on $I_{-}$, where the particles with angular momenta $l_{\phi_{1}}=0, l_{\phi_{2}} \neq 0$ can stay. The left graph in Fig. 4 shows the intersection of $U$ by $\eta=0$ for $\left(l_{\phi_{1}}, l_{\phi_{2}}\right)=(0,16)$ in the same choice of the parameters $\left(k_{1}, k_{2}, l_{1}\right)$. The right figure shows the contours of $U$. At the horizon $(\eta, \xi)=(0,0), U$ diverges to $-\infty$ due to the gravitational force. The potential $U$ seems to have a local minimum but this is not the case because the Hessian gets negative. This shows that there exist unbounded orbits of massless particles on $I_{-}$. As seen from the right figure, a negative local minimum exists at the place far from the axis rather than on it. For massless particles, there are no stable bound orbits because the red curve $U=0$ is not closed, whereas for massive particles with the mass $m$, there are stable bound orbits within the region $U \leq-m^{2} / E^{2}$. 

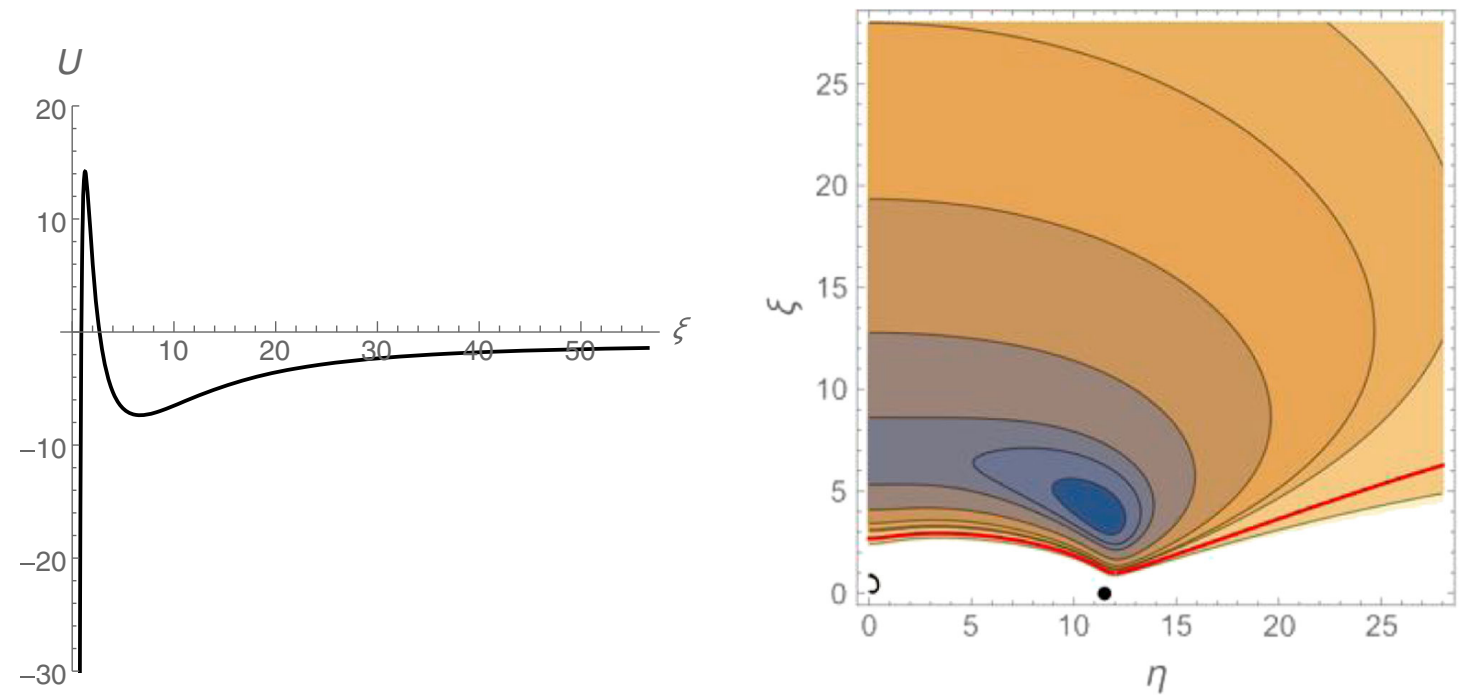

FIG. 4. The left figure shows the effective potential $U$ on $I_{-}(\eta=0, \xi>0)$ for $\left(k_{1}, k_{2}, l_{1}\right)=(0,10,1)$ and $\left(l_{\phi_{1}}, l_{\phi_{2}}\right)=(0,16)$. The right figure shows the corresponding two-dimensional plot of $U$.

Let us discuss whether there can exist stable bound orbits at the other infinity $z \rightarrow-\infty(\eta=0, \xi \rightarrow \infty)$ on the $z$ axis. To do so, we expand the potential $U$ at $z \rightarrow-\infty$ as

$$
U \simeq-1+\frac{U_{-\infty}^{(1)}}{z}+\frac{U_{-\infty}^{(2)}}{z^{2}}
$$

where the constants $U_{-\infty}^{(1)}$ and $U_{-\infty}^{(2)}$ are denoted, respectively, by

$$
U_{-\infty}^{(1)}=\frac{-l_{\phi_{2}}^{2}+16 k_{2}^{2}+8 l_{1}}{4},
$$

$$
U_{-\infty}^{(2)}=\frac{-\left(10 k_{2}^{2}+9 l_{1}\right) l_{\phi_{2}}^{2}+\left(48 k_{2}^{3}+72 k_{2} l_{1}\right) l_{\phi_{2}}-32 k_{2}^{4}-192 k_{2}^{2} l_{1}-24 l_{1}^{2}}{24} .
$$

The inequalities $0<U_{-\infty}^{(1)} \ll 1$ and $U_{-\infty}^{(2)}>0$ are necessary for $U$ to have a local minimum at the infinity $z \rightarrow-\infty$. The condition $0<U_{-\infty}^{(1)} \ll 1$ can be denoted by $l_{\phi_{2}}= \pm 2 \sqrt{2\left(2 k_{2}^{2}+l_{1}\right)} \mp \epsilon(0<\epsilon \ll 1)$, and the leading term of $U_{-\infty}^{(2)}$ can be written as

$$
U_{-\infty}^{(2)} \simeq-\frac{2}{3}\left(12 k_{2}^{4}+26 l_{1} k_{2}^{2}+6 l_{1}^{2} \mp 3\left(2 k_{2}^{3}+3 k_{2} l_{1}\right) \sqrt{2\left(2 k_{2}^{2}+l_{1}\right)}\right)+\mathcal{O}(\epsilon) .
$$

From the leading terms, we find that $U_{-\infty}^{(2)}<0$ for any parameter $l_{1}, k_{2}$, which cannot satisfy the necessary condition for the existence of stable bound orbits in the asymptotic region $z \rightarrow-\infty$, at least, on the $z$ axis.

Moreover, let us expand $U$ near the horizon $z=0$ as

$$
U \simeq-\frac{l_{1}^{2}}{z^{2}}+\frac{2 l_{1}\left(8 k_{2}^{2}-3 l_{1}\right)}{\left(2 k_{2}^{2}-3 l_{1}\right) z}+\mathcal{O}(1)
$$

and then we find that the first and second terms are negative, which is due to the effect of the attraction by the horizon. From Fig. 4, $U$ does not make a local minimum on the $z$ axis.

\section{3. $I_{1}$}

Finally, we consider the potential $U$ on the interval $I_{1}$, where the particles with the angular momenta of the special ratio of $l_{\phi_{1}} / l_{\phi_{2}}=-2$ are allowed to stay. Figure 5 shows the typical behavior of the potential $U$ with a local minimum for the same set of parameters $\left(k_{1}, k_{2}, l_{1}\right)=$ $(0,10,1)$ and $\left(l_{\phi_{1}}, l_{\phi_{2}}\right)=(36,-18)$. Near the horizon at $(\eta, \xi)=(0,0), U$ increases by the effect of the centrifugal force, while as closer to the horizon, the potential diverges to $-\infty$ by the stronger effect of the gravitational force. On the other hand, near the other center $(\eta, \xi)=\left(\eta_{2}, 0\right)$, due to the effect of the centrifugal force, the potential again increases rapidly and then diverges to $\infty$. As a result, there is necessarily a negative local minimum somewhere 

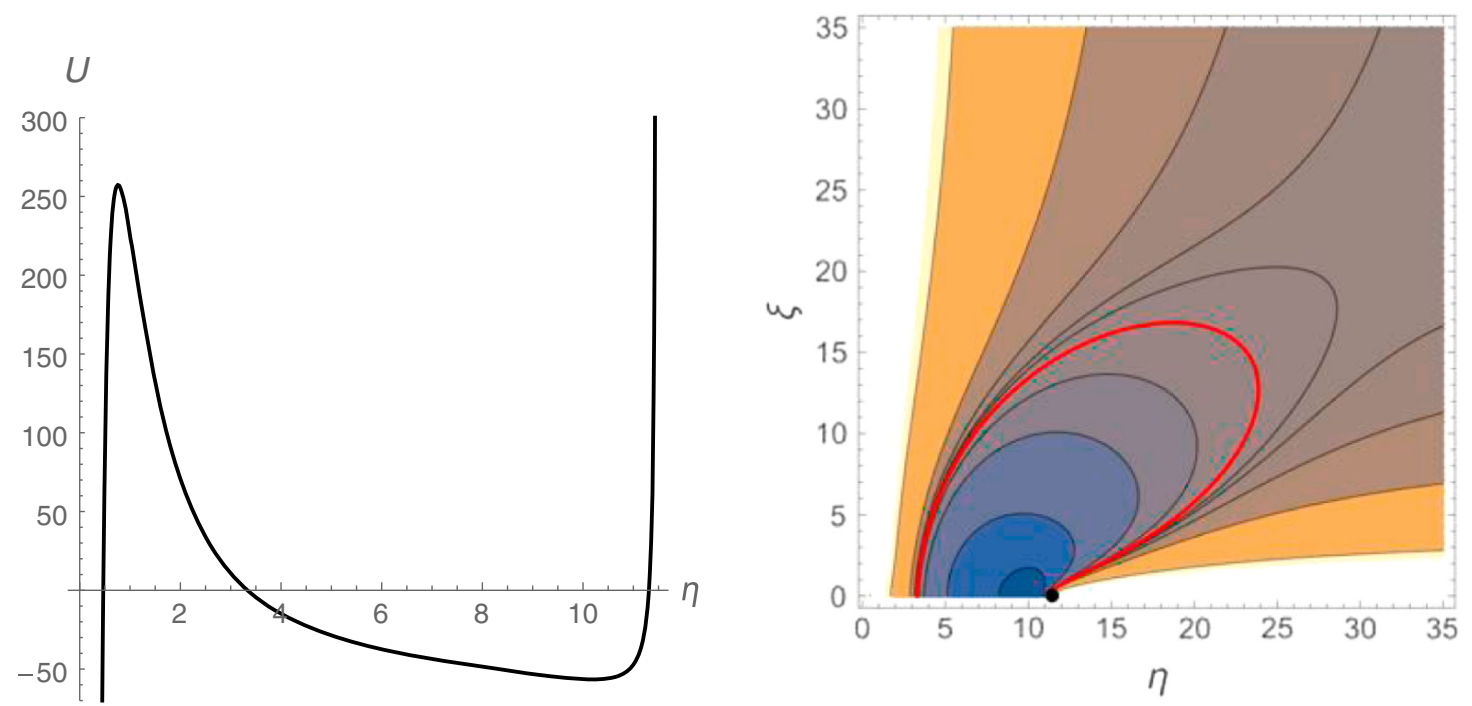

FIG. 5. The left figure shows the effective potential $U$ on $I_{1}\left(0<\eta<\eta_{2}=11.4 \ldots, \xi=0\right)$ for $\left(k_{1}, k_{2}, l_{1}\right)=(0,10,1)$ and $\left(l_{\phi_{1}}, l_{\phi_{2}}\right)=(-72,36)$. The right figure shows the corresponding two-dimensional plot of $U$.

between the horizon $(\eta, \xi)=(0,0)$ and the center $(\eta, \xi)=$ $\left(\eta_{2}, 0\right)$ on the $\eta$ axis. This is the reason why there exist stable bound orbits of massless particles as well as massive particles in a black lens spacetime.

\section{B. A black lens with the topology $L(3,1)$}

Next, let us consider the black lens with the topology of $L(3,1)$. The $z$ axis of the Gibbons-Hawking space is composed of the four intervals: $I_{-}=\{(\eta, \xi) \mid \eta=0, \xi>0\}$, $I_{1}=\left\{(\eta, \xi) \mid 0<\eta<\eta_{2}, \xi=0\right\}, \quad I_{2}=\left\{(\eta, \xi) \mid \eta_{2}<\eta<\right.$ $\left.\eta_{3}, \xi=0\right\}$ and $I_{+}=\left\{(\eta, \xi) \mid \eta>\eta_{3}, \xi=0\right\}$. On $I_{+}$and $I_{-}$, only the particles with the angular momenta of $l_{\phi_{2}}=0$ and $l_{\phi_{1}}=0$, respectively, are allowed to stay there, whereas only ones with the angular momenta of the ratio $l_{\phi_{1}} / l_{\phi_{2}}=$ $-(4-i) /(3-i)$ are allowed to stay on $I_{i}(i=1,2)$.

\section{1. $I_{+}$}

First, let us see the shape of the potential $U$ on $I_{+}$. The left figure of Fig. 6 shows the shape of the typical effective potential with a negative local minimum on the $z$ axis for the particles with the angular momenta of $\left(l_{\phi_{1}}, l_{\phi_{2}}\right)=(5000,0)$ for $\left(k_{1}, k_{2}, k_{3}, l_{1}\right)=(0,10,-50,1)$. The right figure shows

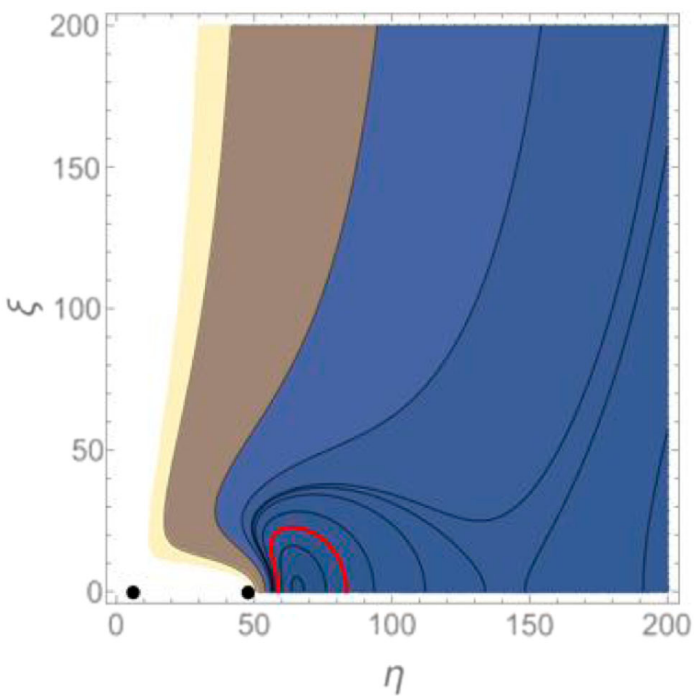

FIG. 6. The left figure shows the potential $U$ for the particles with the angular momenta of $\left(l_{\phi_{1}}, l_{\phi_{2}}\right)=(5000,0)$ for $\left(k_{1}, k_{2}, k_{3}, l_{1}\right)=(0,10,-50,1)$. The right figure shows the contours of $U$ for the particles with the same angular momenta. The red closed curve $U=0$ separates the two-dimensional $(\eta, \xi)$ plane into two regions $U>0$ and $U<0$. The two black points in the right figure denote the points $\left(\eta_{2}, 0\right)$ and $\left(\eta_{3}, 0\right)$, where $\eta_{2}=6.36 \ldots, \eta_{3}=47.91 \ldots$ and the origin $(0,0)$ corresponds to the horizon. 
the contours of $U$ for the particles with the same angular momenta. The closed red curve in this figure, which corresponds to $U=0$, separates the two-dimensional $(\eta, \xi)$ plane into two regions, the outer region $U>0$ and the inner region $U<0$. As can be read off from the contours, $U$ has a negative local minimum at a certain point $(\eta, \xi)=$ $\left(\eta_{\mathrm{m}}, 0\right)$ on the $\eta$ axis $\left(z_{\mathrm{m}}\right.$ on the $z$ axis $)$, where $\eta_{\mathrm{m}}\left(=2 \sqrt{z_{\mathrm{m}}}\right)$ is a certain constant satisfying $\eta_{2}<\eta_{\mathrm{m}}$. At the point $U_{, \eta}=U_{, \xi}=0, \operatorname{det}\left(H_{i j}\right)>0$ and $\operatorname{Tr}\left(H_{i j}\right)>0$. Therefore, both massive and massless particles inside the red curve are stably bounded in the finite region $U \leq-m^{2} / E^{2}$ and $U \leq 0$, respectively. This means that there are stable bound orbits for massive and massless particles.

Moreover, let us consider whether there exist stable bound orbits at infinity $z \rightarrow \infty$. To this end, let us expand the potential $U$ at $z \rightarrow \infty$ as

$$
U \simeq-1+\frac{U_{\infty}^{(1)}}{z}+\frac{U_{\infty}^{(2)}}{z^{2}},
$$

where

$$
U_{\infty}^{(1)}=\frac{l_{\phi_{1}}^{2}-16\left(k_{2}^{2}+k_{2} k_{3}+k_{3}^{2}\right)-8 l_{1}}{4},
$$

and $U_{\infty}^{(2)}$ can be written as a certain quadratic equation for $l_{\phi_{1}}$. It turns out from this asymptotic behavior that the inequalities $0<-U_{\infty}^{(1)} \ll 1$ and $U_{\infty}^{(2)}>0$ are required so that $U$ has a local minimum at infinity because the local minimum is at $z \simeq-2 U_{\infty}^{(2)} / U_{\infty}^{(1)}$. The former condition $0<-U_{\infty}^{(1)} \ll 1$ can be denoted in the form of $l_{\phi_{1}}= \pm 2 \sqrt{4\left(k_{2}^{2}+k_{2} k_{3}+k_{3}^{2}\right)+2 l_{1}} \mp \epsilon(0<\epsilon \ll 1), \quad$ under whose condition the sign of $U_{\infty}^{(2)}$ is determined by the leading term of $U_{\infty}^{(2)}$ in the expansion $U_{\infty}^{(2)} \simeq U_{\infty 0}^{(2)}+\mathcal{O}(\epsilon)$. However, we can see numerically that the region $U_{\infty 0}^{(2)}>0$ does not overlap the parameter region $\mathcal{D}$, which means that there are no stable bound orbits on the $z$ axis in the asymptotic region $z \rightarrow \infty$.

Next we study the existence of stable bound orbits near the end point $z=z_{3}$ of $I_{+}$, near which the potential behaves as

$$
U \simeq \frac{U_{3+}^{(-1)}}{z-z_{3}}+U_{3+}^{(0)}+U_{3+}^{(1)}\left(z-z_{3}\right)
$$

where

$$
U_{3+}^{(-1)}=-\frac{\left[\left(k_{2}-k_{3}\right)^{3} z_{3}+\left(\left(l_{\phi_{1}}-3 k_{2}-6 k_{3}\right) z_{3}-3 l_{1} k_{3}+3 k_{3}^{3}\right) z_{32}\right]^{2}}{4 z_{3} z_{32}\left[\left(k_{2}-k_{3}\right)^{2} z_{3}+\left(l_{1}-3 k_{3}^{2}+z_{3}\right) z_{32}\right]} .
$$

We find numerically that $U_{3+}^{(-1)}>0$ is always satisfied within the parameter region $\mathcal{D}$, so that the potential diverges to $\infty$ at $z=z_{3}$. Moreover, when $0<U_{3+}^{(-1)} \ll 1, U_{3+}^{(1)}>0$ and $U_{3+}^{(0)}+2 \sqrt{U_{3+}^{(-1)} U_{3+}^{(1)}}<0, U$ has a negative local minimum near $z=z_{3}$ on the $z$ axis.

Let us consider the geodesic motion of the massless particles with zero-energy limit $E \rightarrow 0$, in which case it is more convenient to use the potential $U^{\prime}$ rather than $U$. In Fig. 7, the left figure shows the typical shape of the effective potential $U^{\prime}$ for the particles with the angular momenta $L_{\phi_{2}} \neq 0$, where we put $\left(k_{1}, k_{2}, k_{3}, l_{1}\right)=$ $(0,10,-50,1)$ and $\left(L_{\phi_{1}}, L_{\phi_{2}}\right)=(1,0)$. The right figure shows the contours of $U^{\prime}$ for the particles with the same angular momenta. The potential $U^{\prime}$ has a zero local minimum on the evanescent ergosurface, where massless particles with zero energy are stably trapped.

\section{2. $I_{-}$}

Next, let us see the shapes of the effective potential on $I_{-}$. The left figure of Fig. 8 shows the typical shape of the potential $U$ with a local minimum, where this corresponds to the particles with the angular momenta of $\left(l_{\phi_{1}}, l_{\phi_{2}}\right)=(0,50)$ for $\quad\left(k_{1}, k_{2}, k_{3}, l_{1}\right)=(0,10,-50,1)$. The right figure shows the contours of $U$ for the particles with the same angular momenta. The red curve corresponding to $U=0$ is not closed. The potential $U$ does not have a local minimum on the $\xi$ axis (on the $z$ axis corresponding to $z<0$ ), but as is seen from the right contour plots of $U$, there are the closed contours $U=$ $U_{0}\left(U_{0}<0\right)$ which cross the $\xi$ axis. Therefore, massive particles crossing the $\xi$ axis inside the region $U \leq U_{0}$ turn out to be stably bounded. Furthermore, massive particles can also stably bounded inside closed contours apart from the $\xi$ axis.

We discuss the existence of stable bound orbits at the infinity $z \rightarrow-\infty$ on the $z$ axis. Similarly we expand the potential $U$ at $z \rightarrow-\infty$ as

$$
U \simeq-1+\frac{U_{-\infty}^{(1)}}{z}+\frac{U_{-\infty}^{(2)}}{z^{2}},
$$

where

$$
U_{-\infty}^{(1)}=\frac{-l_{\phi_{2}}^{2}+16\left(k_{2}^{2}+k_{2} k_{3}+k_{3}^{2}\right)+8 l_{1}}{4},
$$



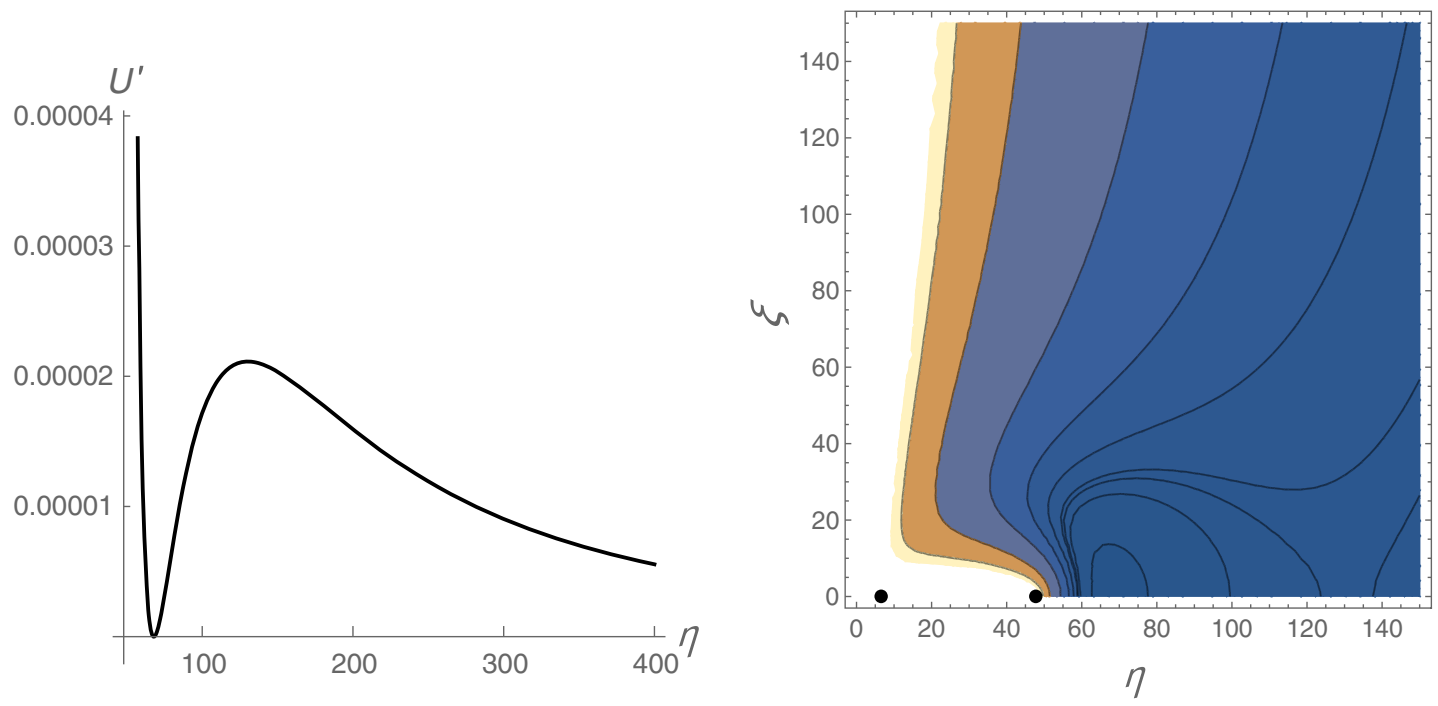

FIG. 7. The left figure shows the potential $U^{\prime}$ under the parameter setting $\left(k_{1}, k_{2}, k_{3}, l_{1}\right)=(0,10,-50,1)$ and angular momenta $\left(L_{\phi_{1}}, L_{\phi_{2}}\right)=(1,0)$. The right figure shows the contours of $U^{\prime}$ for the particles with the same angular momenta. The potential has a local minimum, whose values are zero on the evanescent ergosurfaces.

and $U_{-\infty}^{(2)}$ is a certain quadratic equation for $l_{\phi_{2}}$. The inequalities $0<U_{-\infty}^{(1)} \ll 1$ and $U_{-\infty}^{(2)}>0$ are the necessary conditions for $U$ to have a local minimum at the infinity. The condition $0<U_{-\infty}^{(1)} \ll 1$ can be denoted by $l_{\phi_{2}}=$ $\pm 2 \sqrt{2\left(2 k_{2}^{2}+2 k_{2} l_{3}+2 k_{3}^{2}+l_{1}\right)} \mp \epsilon(0<\epsilon \ll 1), \quad$ and then the leading term of $U_{-\infty}^{(2)}$ can be written as $U_{-\infty}^{(2)} \simeq U_{-\infty 0}^{(2)}+\mathcal{O}(\epsilon)$. However, we can see numerically that the region $U_{-\infty 0}^{(2)}>0$ does not have an overlap region with the parameter region $\mathcal{D}$, which means that there are no stable bound orbits in the asymptotic region $z \rightarrow-\infty$ on the $z$ axis.

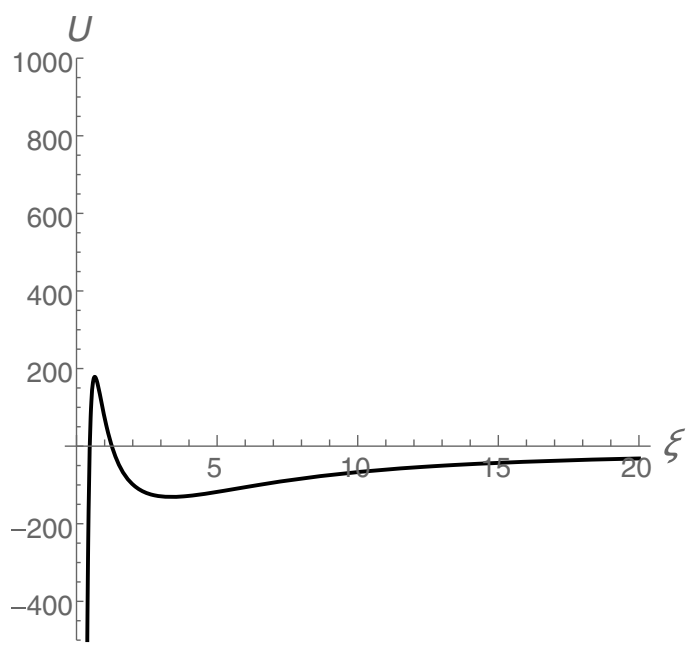

Near the horizon $z=0, U$ behaves as

$$
U \simeq-\frac{l_{1}^{2}}{z^{2}}+\frac{2 l_{1}\left(z_{2} z_{3}+k_{2}^{2} z_{3}+k_{3}^{2} z_{2}\right)}{z_{2} z_{3} z} .
$$

The negativity of the first and second terms makes $U$ diverge to $-\infty$ at the horizon $z=z_{1}(=0)$, which is a pure effect of gravity.

Moreover, to consider the zero-energy limit for particles with the angular momenta $L_{\phi_{1}}=0$, let us see Fig. 9. The left figure shows the effective potential $U^{\prime}$ for the particles with $\left(L_{\phi_{1}}, L_{\phi_{2}}\right)=(0,1)$ under the parameter setting $\left(k_{1}, k_{2}, k_{3}, l_{1}\right)=(0,10,-50,1)$. The right figure shows

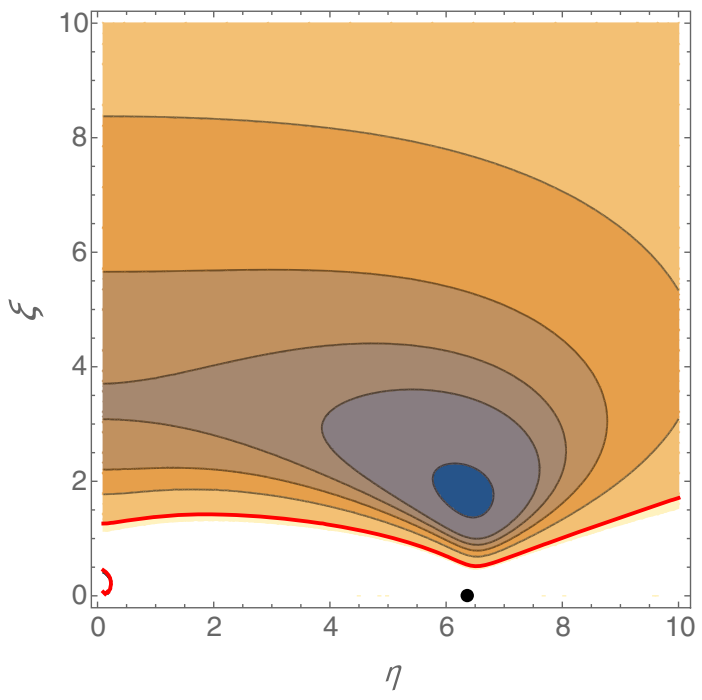

FIG. 8. The left figure shows the effective potential $U$ for the particles with the angular momenta of $\left(l_{\phi_{1}}, l_{\phi_{2}}\right)=(0,50)$ for $\left(k_{1}, k_{2}, k_{3}, l_{1}\right)=(0,10,-50,1)$. The right figure shows the contours of $U$ for the particles with the same angular momenta. The red curve corresponding to $U=0$ is not closed. 

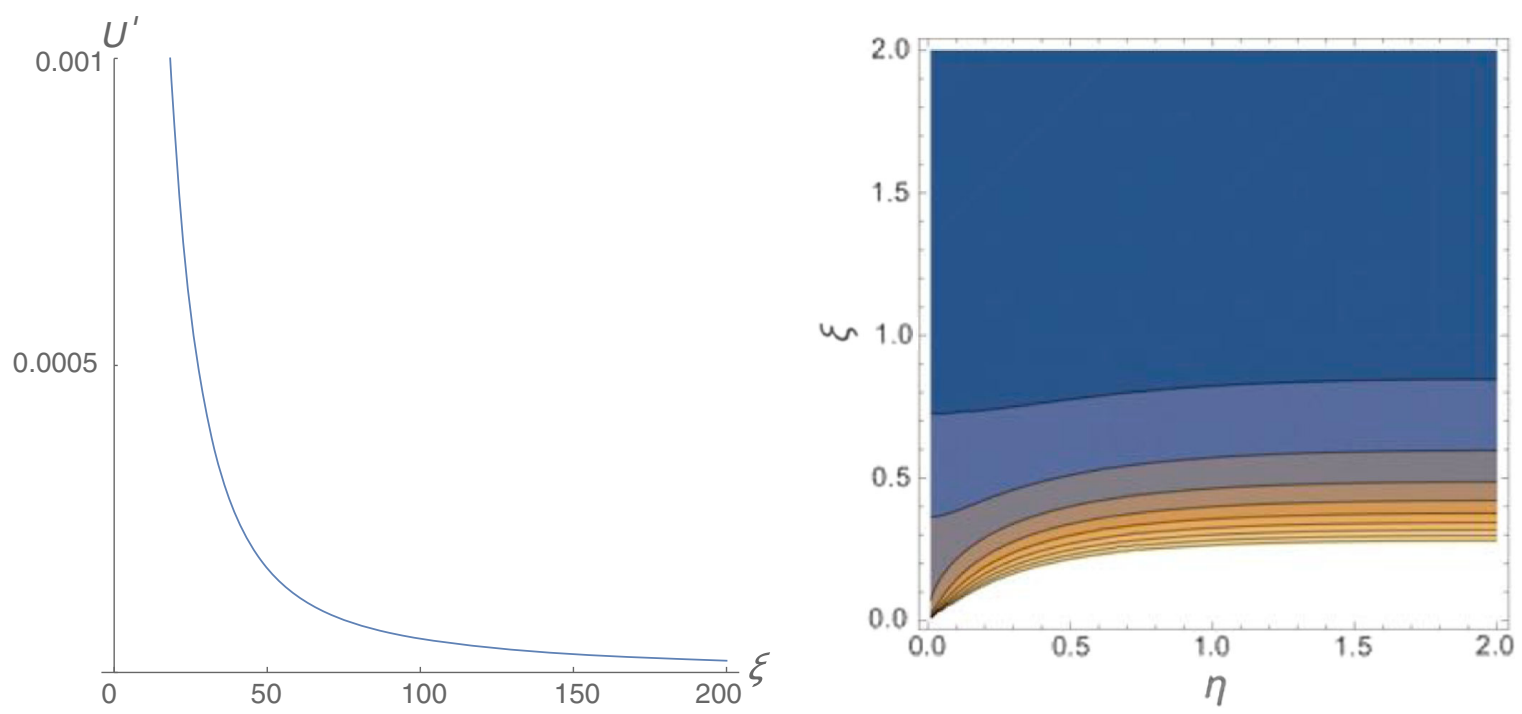

FIG. 9. The left figure shows the effective potential $U^{\prime}$ for the particles with the angular momenta $\left(L_{\phi_{1}}, L_{\phi_{2}}\right)=(0,1)$ under the parameter setting $\left(k_{1}, k_{2}, k_{3}, l_{1}\right)=(0,10,-50,1)$. The right figure shows the contours of $U^{\prime}$ for the particles with the same angular momenta. The potential does not have a local minimum for the absence of evanescent ergosurface on the $z$ axis.

the contours of $U^{\prime}$ for the particles with the same angular momenta. The potential $U^{\prime}$ does not have a local minimum for the absence of evanescent ergosurface on the $z$ axis. Massless particles with zero energy are not stably trapped.

\section{3. $I_{1}$}

Let us discuss the existence of stable bound orbits at the interval $I_{1}$ on the $z$ axis. When we expand the potential $U$ at $z=z_{2}$ as

$$
U \simeq \frac{U_{2-}^{(1)}}{z-z_{2}}+\mathcal{O}(1)
$$

the signature of $U_{2-}^{(1)}$ does not depend on angular momenta $l_{\phi_{1}}$ and $l_{\phi_{2}}$ of particles, and $U_{2-}^{(1)}<0$ is satisfied within the whole parameter region. Therefore $U$ diverges to $\infty$ at $z=z_{2}$ on $I_{1}$. On the other hand, near the horizon $z=0, U$ behaves as

$U \simeq-\frac{l_{1}^{2}}{z^{2}}-\frac{2 l_{1}\left[\left(k_{3}^{2}+z_{3}\right) z_{2}+k_{2}^{2} z_{3}\right]}{z_{2} z_{3} z}+U_{1+}^{(0)}+U_{1+}^{(1)} z$.

The negativity of the first and second terms makes $U$ diverge to $-\infty$ at the horizon $z=z_{1}(=0)$, which is a pure effect of gravity. In order that $U$ has a local minimum near the horizon, $U$ must have a local maximum because $U$ diverges to $\infty$ at $z=z_{2}$. Hence, $U_{1+}^{(1)}$ must be negative, which can be realized for sufficiently large $l_{\phi_{1}}=$ $\left|-3 l_{\phi_{2}} / 2\right|$ because for $\left|l_{\phi_{2}}\right| \rightarrow \infty$,
$U_{1+}^{(1)} \simeq-\frac{\left(3 k_{3}^{2}+l_{1}\right) z_{2}+\left(3 k_{2}^{2}+l_{1}\right) z_{3}+3 z_{2} z_{3}}{16 l_{1} z_{2} z_{3}} l_{\phi_{2}}^{2}<0$.

Figure 10 shows the typical shape of the effective potential $U$. The left figure shows the effective potential on the $\eta$ axis $\left(\eta_{1} \leq \eta \leq \eta_{2}\right)$ [on the $z$ axis $\left(0 \leq z \leq z_{2}\right)$ ] for the particles with the angular momenta $\left(l_{\phi_{1}}, l_{\phi_{2}}\right)=(-1500,1000)$ under the parameter setting $\left(k_{1}, k_{2}, k_{3}, l_{1}\right)=(0,10,-50,1)$ and the right figure shows the contours of $U$ for the particles with the same angular momenta. One can see from these figures that $U$ has a negative local minimum on $I_{1}$ and inside the red curve $U=0$, both massive and massless particles are stably bounded (in the finite region $U \leq-m^{2} / E^{2}$ and $U \leq 0$, respectively).

To consider the geodesic motion of the massless particles with zero-energy limit $E \rightarrow 0$, let us see Fig. 11. The left figure shows the typical effective potential $U^{\prime}$ for the particles with the angular momenta $L_{\phi_{1}} / L_{\phi_{2}}=-3 / 2$ under the parameter setting $\left(k_{1}, k_{2}, k_{3}, l_{1}\right)=(0,10,-50,1)$ and $\left(L_{\phi_{1}}, L_{\phi_{2}}\right)=(3,-2)$. The right figure shows the contours of $U^{\prime}$ for the particles with the same angular momenta. The potential has a zero local minimum on the evanescent ergosurface, where massless particles with zero energy are stably trapped.

\section{4. $I_{2}$}

Finally, let us discuss the existence of stable bound orbits on $I_{2}$. This type of an interval does not exist for the black lens with $L(2,1)$ topology. When we expand the potential $U$ at $z=z_{2}, z_{3}$ as 

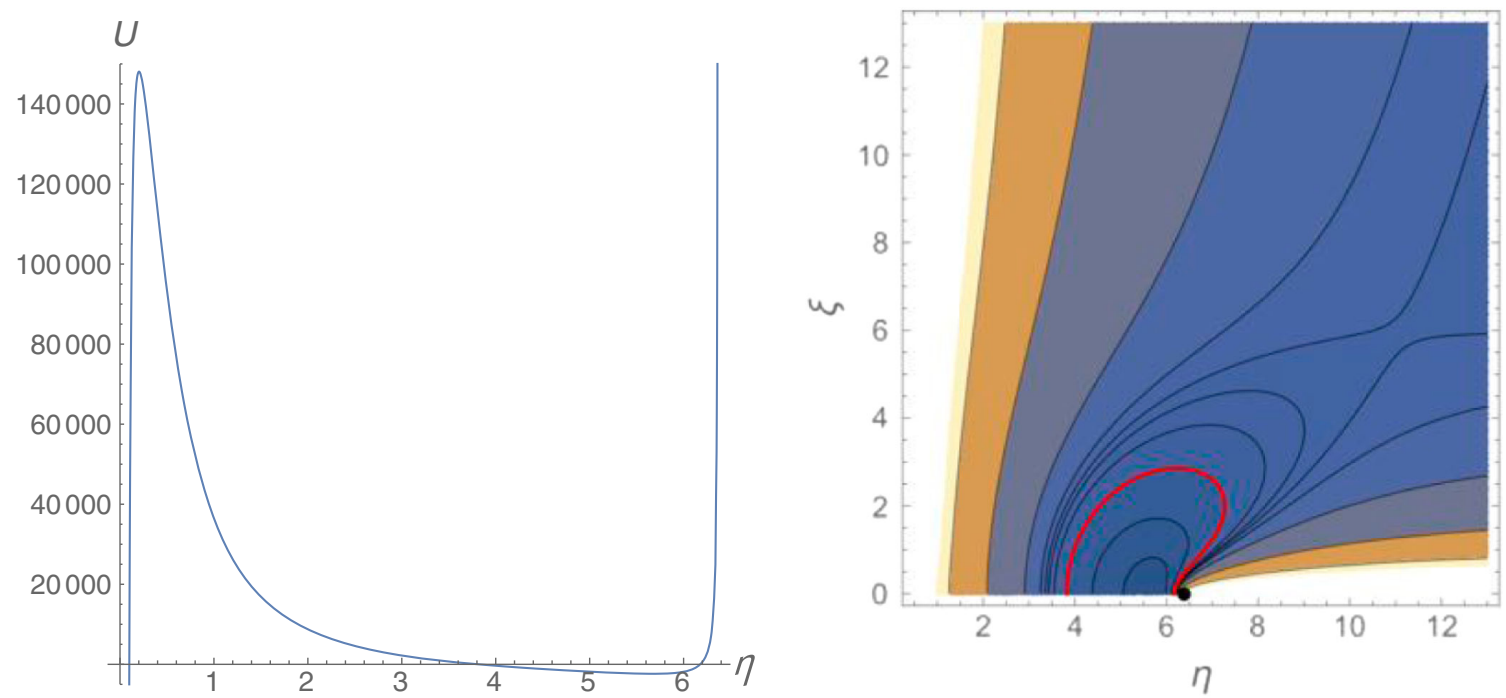

FIG. 10. The left figure shows the effective potential $U$ for the particles with the angular momenta $\left(l_{\phi_{1}}, l_{\phi_{2}}\right)=(-1500,1000)$ under the parameter setting $\left(k_{1}, k_{2}, k_{3}, l_{1}\right)=(0,10,-50,1)$. The right figure shows the contours of $U$ for the particles with the same angular momenta.

$$
U \simeq \frac{U_{i}^{(1)}}{z-z_{i}}(i=2+, 3-)
$$

regardless of the angular momenta $l_{\phi_{1}}$ and $l_{\phi_{2}}$ of particles, $U_{2-}^{(1)}$ and $U_{3+}^{(1)}$ are positive and negative, respectively, within the parameter region $\mathcal{D}$. Therefore $U$ diverges to $\infty$ at $z=z_{2}$ and $z=z_{3}$ on $I_{2}$. As is already expected, $U$ has a local minimum on $I_{2}$.

Figure 12 shows the typical shape of the effective potential $U$. The left figure shows the effective potential on the $\eta$ axis $\left(\eta_{2} \leq \eta \leq \eta_{3}\right)$ [on the $z$ axis $\left(z_{2} \leq z \leq z_{3}\right)$ ] for the particles with the angular momenta $\left(l_{\phi_{1}}, l_{\phi_{2}}\right)=(-2000,1000)$ under the parameter setting $\left(k_{1}, k_{2}, k_{3}, l_{1}\right)=(0,10,-50,1)$ and the

right figure shows the contours of $U$ for the particles with the same angular momenta. One can see from these figures that $U$ has a negative local minimum on $I_{2}$ and inside the red curve $U=0$, both massive and massless particles are stably bounded (in the finite region $U \leq-m^{2} / E^{2}$ and $U \leq 0$, respectively).

Moreover, to consider the zero-energy limit for particles with the angular momenta $L_{\phi_{1}} / L_{\phi_{2}}=-2$, let us see Fig. 13. The left figure shows the effective potential $U^{\prime}$ for the particles with $\left(L_{\phi_{1}}, L_{\phi_{2}}\right)=(-2,1)$ under the parameter setting $\left(k_{1}, k_{2}, k_{3}, l_{1}\right)=(0,10,-50,1)$. The right figure shows the contours of $U^{\prime}$ for the particles with the same angular momenta. The potential has two
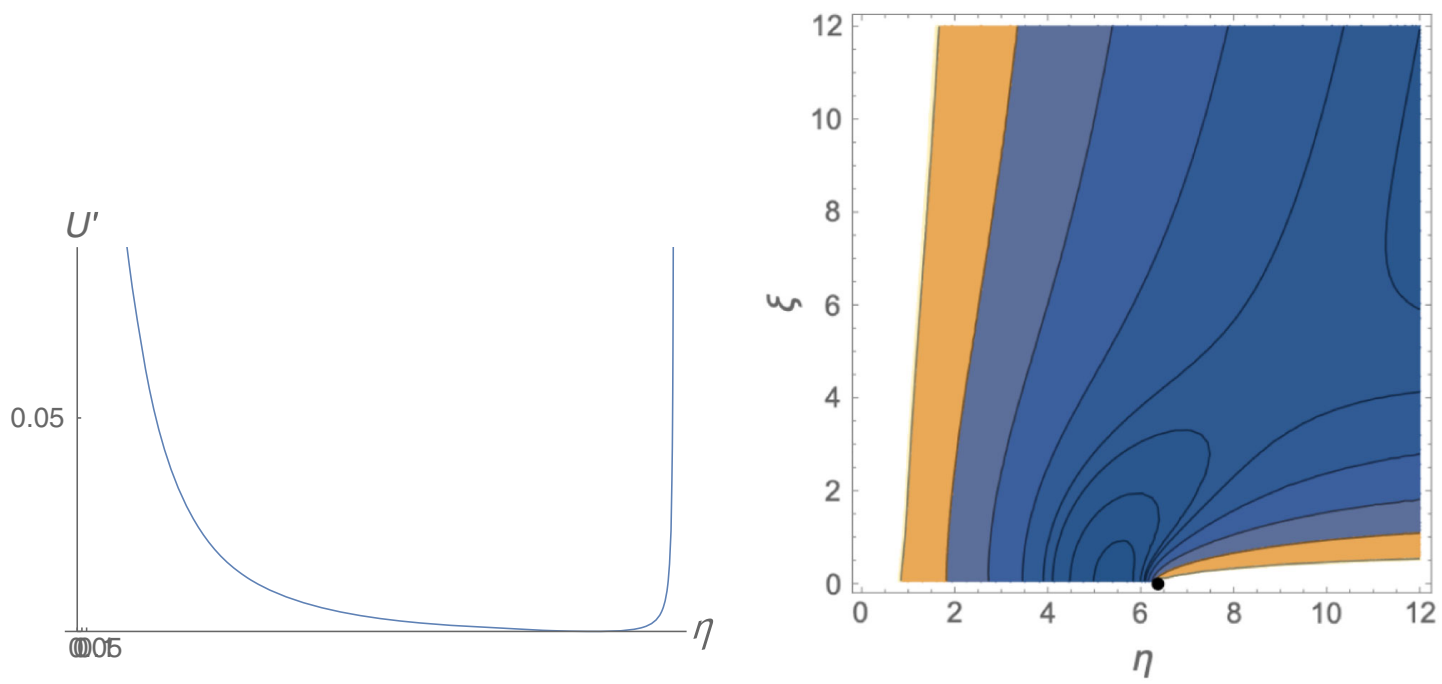

FIG. 11. The left figure shows the typical effective potential $U^{\prime}$ for $\left(k_{1}, k_{2}, k_{3}, l_{1}\right)=(0,10,-50,1)$ and $\left(l_{\phi_{1}}, l_{\phi_{2}}\right)=(3,-2)$. The right figure shows the contours of $U^{\prime}$ for the particles with the same angular momenta. The potential has a local minimum whose values are zero on the evanescent ergosurface. 

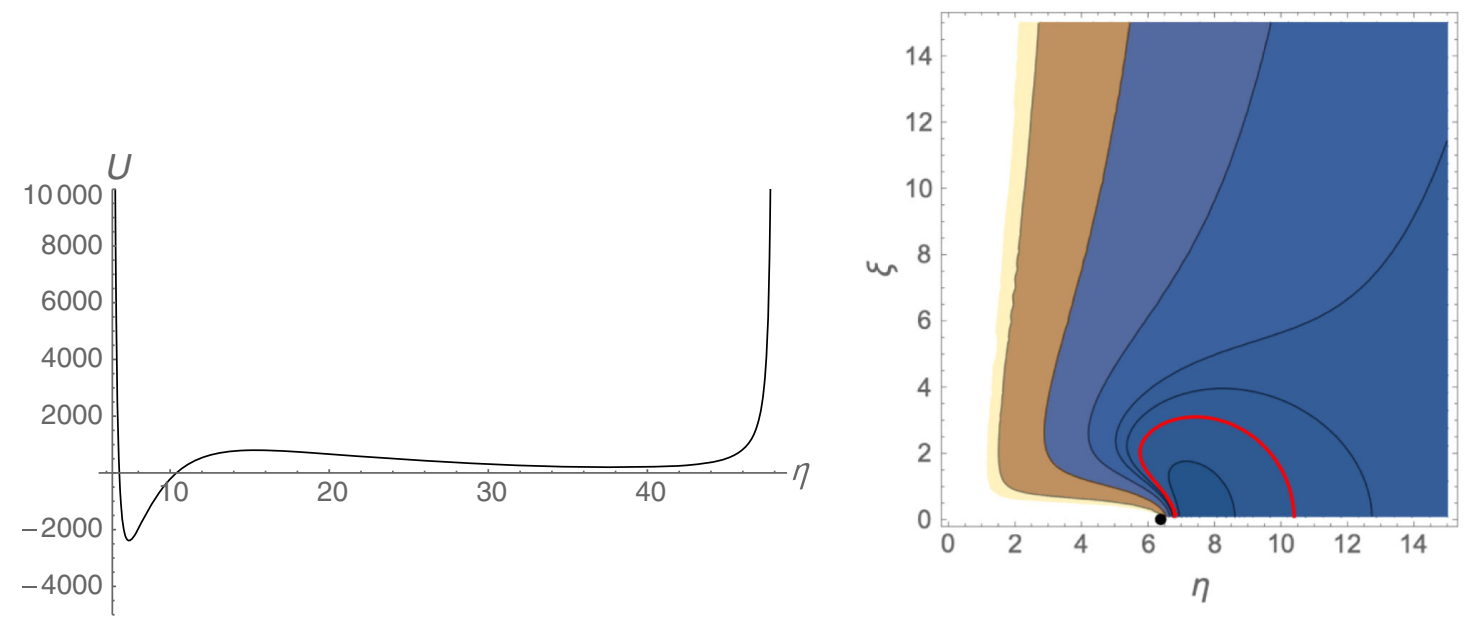

FIG. 12. The left figure shows the effective potential $U$ for the particles with the angular momenta $\left(l_{\phi_{1}}, l_{\phi_{2}}\right)=(-2000,1000)$ under the parameter setting $\left(k_{1}, k_{2}, k_{3}, l_{1}\right)=(0,10,-50,1)$. The right figure shows the contours of $U$ for the particles with the same angular momenta.
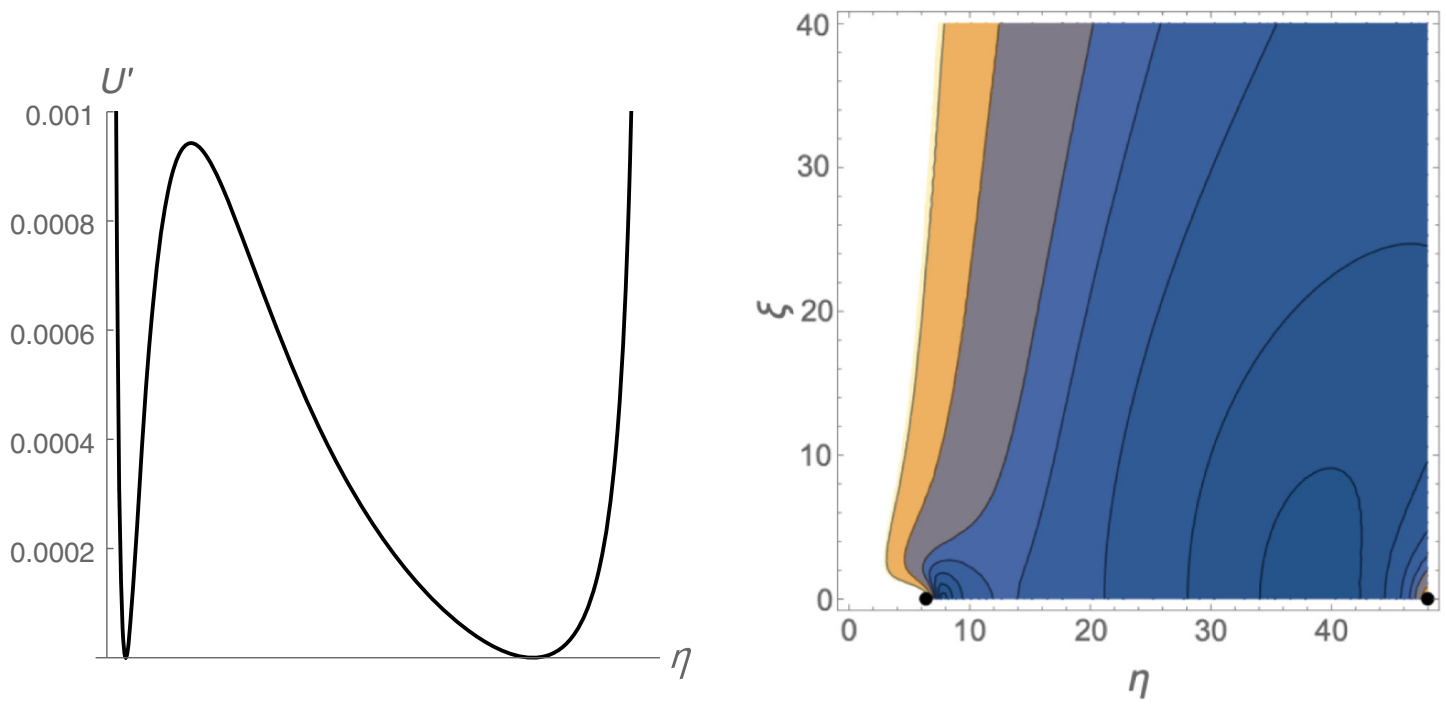

FIG. 13. The left figure shows the effective potential $U^{\prime}$ for the particles with the angular momenta $\left(L_{\phi_{1}}, L_{\phi_{2}}\right)=(-2,1)$ under the parameter setting $\left(k_{1}, k_{2}, k_{3}, l_{1}\right)=(0,10,-50,1)$. The right figure shows the contours of $U^{\prime}$ for the particles with the same angular momenta. The potential has local minima whose values are zero at the evanescent ergosurfaces.

local minima, whose values are zero at the evanescent ergosurfaces.

\section{SUMMARY AND DISCUSSIONS}

In this paper, we have analyzed the geodesics for the supersymmetric black lenses with $L(2,1)$ and $L(3,1)$ topologies in the five-dimensional minimal supergravity. We have reduced the geodesic motion of particles to a two-dimensional potential problem, and from the twodimensional contour plots of the potential, we have clarified the existence of the stable bound orbits for the massive and massless particles. In the previous work [25] on the black lens with $L(2,1)$ topology, where one-dimensional potential was used, we found the stable bound orbits of particles on the $z$ axis corresponding to two rotational axes ( $\eta$ axis and $\xi$ axis). In this work, we have found that there also exist stable bound orbits in a place away from the $z$ axis as well as on the axis. Moreover, we have discussed the range such that stable bound orbits exist on the $z$ axis and have shown that there exist no stable bound orbits in the asymptotic region $z \rightarrow \pm \infty$, at least, on the $z$ axis. This result suggests that there exists the outermost stable circular orbit as the boundary of stable circular orbits.

In particular, we have also discussed the geodesic motion for massless particles with zero energy. We have proved that for such particles, the potential always has a local minimum at the intersection of the evanescent 
ergosurface and the curve $G\left(\eta, \xi, L_{\phi_{1}}, L_{\phi_{2}}\right)=0$ in the $(\eta, \xi)$ plane, where, in particular, for particles with the angular momenta satisfying either condition $L_{\phi_{2}}=0$ or $J=0$, the intersection exists, at least at the rotational axes. This means that such particles are stably trapped on the geodesics on the evanescent ergosurface, which is exactly consistent with the mathematical result [27], which proved that on an evanescent ergosurface, massless particles with zero energy move along stable trapped null geodesics. As shown in Ref. [27], for the microstate geometry, the existence of evanescent ergosurfaces leads to some nonlinear instability. This result does not apply to the solution with a black hole horizon but the presence of stable bound orbits of particles with zero energy even in a black lens background may exhibit corresponding nonlinear instability.

\section{ACKNOWLEDGMENTS}

This work was supported by the Grant-in-Aid for Scientific Research (C) [JSPS KAKENHI Grant No. 17K05452 (S. T.)] and the Grant-in-Aid for EarlyCareer Scientists [JSPS KAKENHI Grant No. JP19K14715 (T. I.)] from the Japan Society for the Promotion of Science. S. T. is also supported from Toyota Technological Institute Fund for Research Promotion (A).
[1] D. C. Wilkins, Bound geodesics in the Kerr metric, Phys. Rev. D 5, 814 (1972).

[2] F. S. Khoo and Y. C. Ong, Lux in obscuro: Photon orbits of extremal black holes revisited, Classical Quantum Gravity 33, 235002 (2016).

[3] S. R. Dolan and J. O. Shipley, Stable photon orbits in stationary axisymmetric electrovacuum spacetimes, Phys. Rev. D 94, 044038 (2016).

[4] K. Nakashi and T. Igata, Innermost stable circular orbits in the Majumdar-Papapetrou dihole spacetime, Phys. Rev. D 99, 124033 (2019).

[5] K. Nakashi and T. Igata, Effect of a second compact object on stable circular orbits, Phys. Rev. D 100, 104006 (2019).

[6] J. Keir, Slowly decaying waves on spherically symmetric spacetimes and ultracompact neutron stars, Classical Quantum Gravity 33, 135009 (2016).

[7] V. Cardoso, L. C. B. Crispino, C. F. B. Macedo, H. Okawa, and P. Pani, Light rings as observational evidence for event horizons: Long-lived modes, ergoregions and nonlinear instabilities of ultracompact objects, Phys. Rev. D 90, 044069 (2014).

[8] S. Hollands and S. Yazadjiev, Uniqueness theorem for 5dimensional black holes with two axial killing fields, Commun. Math. Phys. 283, 749 (2008).

[9] S. Hollands, J. Holland, and A. Ishibashi, Further restrictions on the topology of stationary black holes in five dimensions, Ann. Inst. Henri Poincaré 12, 279 (2011).

[10] M. 1. Cai and G. J. Galloway, On the topology and area of higher dimensional black holes, Classical Quantum Gravity 18, 2707 (2001).

[11] G. J. Galloway and R. Schoen, A generalization of Hawking's black hole topology theorem to higher dimensions, Commun. Math. Phys. 266, 571 (2006).

[12] R. Emparan and H.S. Reall, A Rotating Black Ring Solution in Five-dimensions, Phys. Rev. Lett. 88, 101101 (2002).

[13] A. A. Pomeransky and R. A. Sen'kov, Black ring with two angular momenta, arXiv:hep-th/0612005.
[14] H. Elvang, R. Emparan, D. Mateos, and H. S. Reall, A Supersymmetric Black Ring, Phys. Rev. Lett. 93, 211302 (2004).

[15] H. K. Kunduri and J. Lucietti, Supersymmetric Black Holes with Lens-Space Topology, Phys. Rev. Lett. 113, 211101 (2014).

[16] S. Tomizawa and M. Nozawa, Supersymmetric black lenses in five dimensions, Phys. Rev. D 94, 044037 (2016).

[17] F. R. Tangherlini, Schwarzschild field in $n$ dimensions and the dimensionality of space problem, Nuovo Cimento 27, 636 (1963).

[18] D. N. Page, D. Kubiznak, M. Vasudevan, and P. Krtous, Complete Integrability of Geodesic Motion in General KerrNUT-AdS Spacetimes, Phys. Rev. Lett. 98, 061102 (2007).

[19] V. P. Frolov and D. Stojkovic, Particle and light motion in a space-time of a five-dimensional rotating black hole, Phys. Rev. D 68, 064011 (2003).

[20] V. P. Frolov, P. Krtous, and D. Kubiznak, Separability of Hamilton-Jacobi and Klein-Gordon equations in general Kerr-NUT-AdS spacetimes, J. High Energy Phys. 02 (2007) 005 .

[21] V. Cardoso, A. S. Miranda, E. Berti, H. Witek, and V. T. Zanchin, Geodesic stability, Lyapunov exponents and quasinormal modes, Phys. Rev. D 79, 064016 (2009).

[22] T. Igata, H. Ishihara, and Y. Takamori, Stable bound orbits around black rings, Phys. Rev. D 82, 101501 (2010).

[23] T. Igata, H. Ishihara, and Y. Takamori, Chaos in geodesic motion around a black ring, Phys. Rev. D 83, 047501 (2011).

[24] T. Igata, H. Ishihara, and Y. Takamori, Stable bound orbits of massless particles around a black ring, Phys. Rev. D 87, 104005 (2013).

[25] S. Tomizawa and T. Igata, Stable bound orbits around a supersymmetric black lens, Phys. Rev. D 100, 124031 (2019).

[26] T. Igata and S. Tomizawa, Stable circular orbits in higherdimensional multi-black hole spacetimes, Phys. Rev. D 102, 084003 (2020).

[27] F. C. Eperon, H. S. Reall, and J. E. Santos, Instability of supersymmetric microstate geometries, J. High Energy Phys. 10 (2016) 031. 